\title{
Perfil epidemiológico dos pacientes do serviço de fisioterapia de um município da região metropolitana de Curitiba/PR
}

\section{Epidemiological profile of physiotherapy patients in a municipality in the metropolitan region of Curitiba/PR}

\begin{abstract}
Alexsandra de Carvalho Ramos ${ }^{1}$, Maricleia Carniel${ }^{2}$, Maria de Fatima Fernandes Vara ${ }^{3}$,
\end{abstract}
Elgison da Luz dos Santos ${ }^{4}$

1. ORCID: https://orcid.org/0000-0002-1451-1624. Fisioterapeuta, Egressa do Curso de Fisioterapia da Faculdade Paranaense, Curitiba, Paraná, Brasil.

E-mail: Alexia.reabilitacaofisioterapia@gmail.com

2. ORCID: https://orcid.org/0000-0002-9753-808X. Fisioterapeuta, Egressa do Curso de Fisioterapia da Faculdade Paranaense, Curitiba, Paraná, Brasil.

E-mail: maricleia.fisio@gmail.com

3. ORCID: https://orcid.org/0000-0002-7563-1857. Fisioterapeuta, Doutoranda em Tecnologia em Saúde (PUCPR), Professora da Faculdade Paranaense, Curitiba, Paraná, Brasil.

E-mail: mfatimafv@gmail.com

4. ORCID: https://orcid.org/0000-0002-7563-1857. Fisioterapeuta, Doutor em Tecnologia em Saúde. Servidor da Secretaria Municipal de Saúde de Rio Branco do Sul/PR. Professor da Faculdade Paranaense e do Centro Universitário Uninter, Curitiba, Paraná, Brasil.

E-mail: elgisantos20@gmail.com

CONTATO: Autor correspondente: Elgison da Luz dos Santos | Endereço: São Lucas, 391 - Itaperuçu-Paraná Telefone: (41) 996738423 E-mail: elgisantos20@gmail.com

\section{RESUMO}

Estudos epidemiológicos favorecem a produção de conhecimento, a tomada de decisão e a formulação de políticas públicas para solucionar problemas de saúde específicos. Este estudo tem como objetivo descrever o perfil epidemiológico dos usuários do setor de fisioterapia de uma cidade da região 
metropolitana de Curitiba/PR. Trata-se de um estudo epidemiológico, descritivo, por meio de análise de prontuários, utilizando-se de um roteiro preestabelecido. Todos os prontuários disponíveis no setor, dos anos 2019 e 2020, foram incluídos. Documentos com dados faltantes ou ilegíveis foram excluídos. Foram analisados 277 prontuários. Destes, o sexo masculino foi o mais prevalente (56\%) e a idade média geral foi de $43 \pm 20,0$ e $52 \pm 17,2$ anos para os gêneros masculino e feminino, respectivamente. $O$ maior percentual de atendimentos ocorreu na área da fisioterapia ortopédica (66,06\%), seguido pela neurológica $(28,51 \%)$ e reumatológica $(6,13 \%)$. A partir dos dados, reforça-se a importância da inserção do profissional fisioterapeuta tanto para a reabilitação desses pacientes como em campanhas de educação em saúde.

DESCRITORES: Perfil de Saúde. Modalidades de Fisioterapia. Pacientes. Epidemiologia.

\section{ABSTRACT}

Epidemiological studies favor the production of knowledge, decision-making, and the formulation of public policies to solve specific health problems. This study aims to describe the epidemiological profile of users of the physical therapy sector of a city in the metropolitan region of Curitiba/PR. This is an epidemiological and descriptive study, through the analysis of medical records, using a pre-established script. All medical records available in the sector, from the years 2019 and 2020, were included. Documents with missing or illegible data were excluded. A total of 277 medical records were analyzed. Of these, males were the most prevalent (56\%) and the overall mean age was $43 \pm 20.0$ and $52 \pm 17.2$ years for male and female genders, respectively. The highest percentage of care occurred in the area of orthopedic physiotherapy $(66.06 \%)$, followed by neurological $(28.51 \%)$ and rheumatologic (6.13\%). From the data, the importance of the insertion of the physiotherapist professional both for the rehabilitation of these patients and in health education campaigns is reinforced.

DESCRIPTORS: Health Profile. Physical Therapy Modalities. Patients. Epidemiology. 


\section{INTRODUÇÃO}

om o avanço tecnológico e aumento da hipocinesia, houve um comprometimento na qualidade de vida da população, ocasionando o surgimento de novas patologias como má postura e obesidade. Também se observa o aumento da violência e acidentes automobilísticos que levam a traumas e lesões diretas. Estes são alguns fatores que favorecem as desordens musculoesqueléticas, cardiovasculares e, consequentemente, perda ou limitação funcional. Então, muitas dessas pessoas acometidas procuram atendimento nos serviços de saúde, sendo que em grande parte a procura ocorre nos estabelecimentos públicos. Em muitos casos, estas pessoas são direcionadas para o setor de reabilitação, que inclui a fisioterapia1.

No final dos anos 80, com o surgimento do Sistema Único de Saúde (SUS), diversas mudanças aconteceram no campo da saúde. Um dos princípios defendidos pelo SUS é o da integralidade que se ampara em ações de promoção, prevenção e recuperação da saúde. A integralidade permite uma visão mais holística do indivíduo levando em conta seu contexto histórico social, político, familiar e ambiental em que vive. Sendo assim, a integralidade se torna individual e coletiva ${ }^{2}$, revelando a necessidade de envolvimento multiprofissional, justificando-se a inserção do fisioterapeuta, com suas atribuições legais de promoção a saúde, desenvolvendo protocolos diferenciados na saúde ${ }^{3}$.

Os níveis de atenção no SUS são classificados em três: primário, secundário e terciário. O nível primário é a porta de entrada do usuário no sistema de saúde e inclui as ações voltadas para medidas preventivas, no que se trata de educação e informações junto às pessoas, grupos ou comunidades. Os pontos de atenção secundários e terciários se diferenciam por suas respectivas densidades tecnológicas. A atenção secundária é formada pelos serviços especializados em nível ambulatorial e hospitalar, com densidade tecnológica intermediária entre a atenção primária e a terciária, enquanto que os pontos de atenção terciários são mais densos tecnologicamente que os pontos de atenção secundários e, por essa razão, tendem a ser mais concentrados espacialmente ${ }^{4}$.

O profissional fisioterapeuta é capacitado para atuar em todos os níveis de atenção à saúde, desde primária até a reabilitação que se caracteriza na atenção terciária. Portanto, o profissional fisioterapeuta não está apenas limitado ao setor de 
cura, mas também deve propor ações de prevenção e educação em saúde, visando uma melhoria na qualidade de vida da população ${ }^{5}$. Cada vez tem havido uma maior preocupação com a formação de um profissional fisioterapeuta com perfil voltado para a atenção básica. Neste campo, o fisioterapeuta tem a responsabilidade de formular ações e prestar serviços de tratamento e reabilitação, controlar os riscos e danos em seu território, prevenir lesões e promover a saúde. Então, para que o conhecimento e a prática do fisioterapeuta visem à saúde ou a qualidade de vida da população, os gestores de saúde devem reconhecer o papel e o potencial deste profissional na equipe multidisciplinar da atenção primária ${ }^{6}$.

A fisioterapia é a ciência que examina atentamente o movimento humano com o propósito de precaver e tratar os distúrbios que possam prejudicar a funcionalidade de um indivíduo 7 . Vale destacar que a fisioterapia é relativamente nova no Brasil, pois foi instituída como profissão de nível superior somente em 1969 por meio da publicação do Decreto-Lei 938/69.

Pelo fato de se desconhecerem, muitas vezes, as causas ou relações que envolvem as lesões de um modo em geral, recorre-se a estudos epidemiológicos que auxiliam e fornecem conhecimentos sobre os fatores determinantes das condições de saúde. Um estudo epidemiológico se caracteriza por estudar a distribuição da população e os níveis de acometimento da saúde. Seu principal objetivo é conhecer fatores instáveis de uma determinada região e contribuir para a proteção, promoção e recuperação da saúde dessa população estudada ${ }^{8}$, bem como conhecer o perfil epidemiológico e as demandas de serviços, favorecendo a adequação das ações e dos serviços de saúde.

$\mathrm{Na}$ área da fisioterapia os estudos epidemiológicos são necessários para identificar as principais patologias que acometem determinada região, reforçar a importância do profissional fisioterapeuta ${ }^{9} \mathrm{e}$, ainda, identificar dados que direcionem este profissional para futuras campanhas de promoção a saúde ${ }^{10}$. Por outro lado, há uma carência de pesquisas voltadas a identificar o perfil epidemiológico na área da fisioterapia e, por isso, novos estudos desta magnitude devem ser estimulados cada vez mais.

Desta forma o presente estudo tem como objetivo descrever o perfil epidemiológico dos usuários do setor de reabilitação fisioterapêutica de uma cidade 
da região metropolitana de Curitiba - Paraná. A motivação em realizar tal investigação para este público é que o setor poderá adequar formas de tratamento, bem como conscientizar a população sobre o melhor aproveitamento do serviço permitindo, indiretamente, aos gestores e profissionais novas estratégias que utilizem os recursos/investimentos em saúde de forma mais apropriada e apresentem maior resolubilidade dos casos.

\section{MÉTODO}

Trata-se de um estudo quantitativo, descritivo, realizado no Centro Municipal de Reabilitação de Rio Branco do Sul/PR, cujas coletas de dados foram realizadas em julho de 2021. A pesquisa descritiva tem como objetivo detalhar as características de uma determinada população, fenômeno ou experiência ${ }^{11}$.

O município onde ocorreu a coleta de dados possui aproximadamente 32.397 habitantes de população total (estimativa IBGE/2019) ${ }^{12}$, e o único atendimento público de fisioterapia ocorre no Centro Municipal de Reabilitação. Desta forma, o setor acolhe encaminhamentos provenientes das Unidades básicas de Saúde, do hospital Municipal e de grandes hospitais da região de Curitiba. O centro de reabilitação conta com um total de 6 profissionais da fisioterapia para atendimento de toda a demanda do município. Devido ao alto fluxo de pacientes com solicitações de fisioterapia, o setor possui grande fila de espera. Apesar de alguns profissionais possuírem cursos de especializações, no setor, todos atendem como generalistas. Portanto, atendem as diferentes áreas conforme a demanda.

Após aprovação no comitê de ética em pesquisa, de acordo com horário de funcionamento do setor de fisioterapia e disponibilidade dos funcionários, os pesquisadores realizaram um agendamento para início da coleta de dados. A coleta aconteceu por meio da análise dos prontuários dos pacientes que iniciaram os atendimentos nos anos de 2019 e 2020 . Foram pré-selecionados e incluídos todos os prontuários referentes a estas datas. Após a seleção, os prontuários foram organizados pelos pesquisadores por ano e ordem alfabética. Para extração dos dados, elaborou-se uma planilha no programa Microsoft ${ }^{\circledR}$ Excel, para anotar as seguintes informações: nome do paciente, idade, sexo, diagnóstico clínico, área de atuação da fisioterapia, número de terapias realizadas e de faltas. Determinou-se 
como critério de exclusão: prontuários inelegíveis, com dados de interesse da pesquisa faltantes/não disponíveis, em segredo de justiça ou, ainda, que por algum motivo o profissional fisioterapeuta responsável pelo paciente não autorizasse o manuseio.

Para a análise e interpretação dos dados utilizou-se o programa Microsoft ${ }^{\oplus}$ Excel. Aplicou-se estatística descritiva simples, mostrando os resultados em número absoluto, percentual e/ou média e desvio padrão (Média \pm DP).

Este estudo foi submetido à apreciação pelo Comitê de Ética em Pesquisa da Universidade Paulista sob o CAAE 46562021.9.0000.5512. Os autores respeitaram a autonomia e a garantia do anonimato dos participantes, assegurando a privacidade dos dados, como rege a Resolução 466/12 do Conselho Nacional de Saúde e suas atualizações. Por tratar-se de um estudo retrospectivo por meio da análise de prontuários, a presente pesquisa teve dispensa da aplicação do Termo de Consentimento Livre e Esclarecido. Ressalta-se que essa pesquisa não provocou a exposição dos indivíduos, uma vez que os dados coletados não forneceram informações suficientes para identificação. Desta maneira, os pesquisadores garantiram a proteção de dados pessoais, a privacidade e a confidencialidade de pacientes, conjugada com a observância de normas deontológicas, referenciais bioéticos e respeito às exigências técnicas.

\section{RESULTADOS}

Foram avaliados todos os prontuários de 2019 ( $n=224)$ e 2020 ( $n=95)$, totalizando 319 documentos. No entanto, foram excluídos 42 (13\%) documentos, sendo 18 (5,6\%) por não constar a informação idade e 24 (7,5\%) por não constar a variável mecanismo de lesão/diagnóstico. Desta forma, a amostra final foi composta de 277 documentos.

A figura 1 apresenta a distribuição quanto ao sexo dos pacientes atendidos no setor. Percebeu-se que o sexo masculino foi o mais prevalente, representando $54 \%$ em ambos os anos, mesmo sendo amostras diferentes. 
Figura 1. Distribuição Quanto ao Sexo dos Pacientes Atendidos no centro de Reabilitação de Rio Branco do Sul - PR (2019 a 2020)

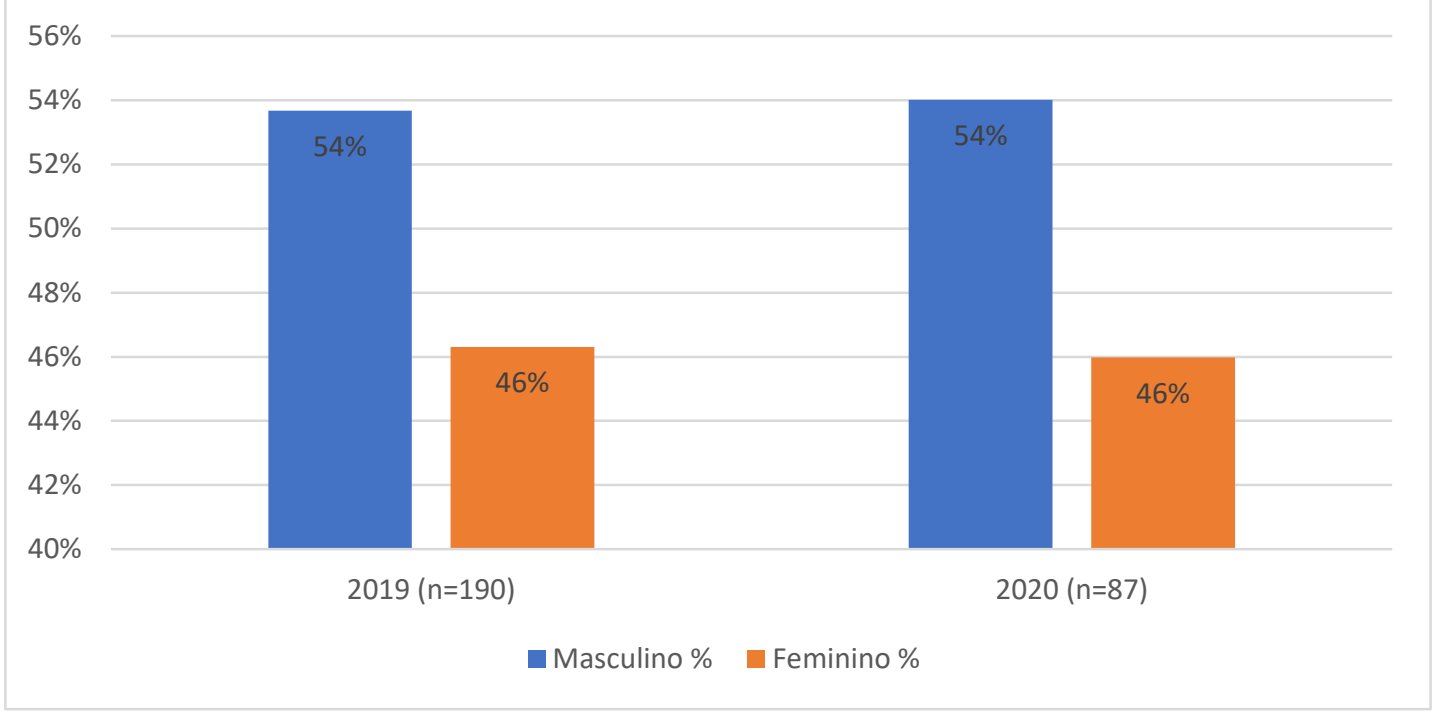

Fonte: Elaborado pelos autores (2021)

A tabela 1 apresenta os dados referentes as áreas (especialidades) da fisioterapia, as quais tiveram maior procura. Apresenta-se também a distribuição entre média de idade e desvio padrão ( $\mathrm{IM} \pm \mathrm{DP})$, considerando as áreas da fisioterapia e o sexo dos pacientes. Para ortopedia-Funcional, o sexo masculino apresentou $40 \pm 16,49$ anos e o público feminino apresentou $48 \pm 15,16$ anos. Para a neuro-funcional, o sexo masculino mostrou a idade média de 47£26,14 anos e o feminino 59 $\pm 20,68$ anos. Na área da reumato-funcional, o masculino apresentou $57 \pm 22,29$ anos e o feminino $60 \pm 11,82$ anos. Na cardiorrespiratória, não houve pacientes do sexo masculino e o público feminino teve a média de 59ะ19,99 anos. Para uroginecologia houve apenas um paciente masculino e um feminino, com idade de 56 e 37 anos, respectivamente. Na oncologia houve apenas um paciente do sexo feminino de 38 anos.

Tabela 1. Distribuição Quanto a área, Sexo, Idade Média e Desvio Padrão dos Pacientes atendidos no Setor de Fisioterapia.

\begin{tabular}{lccccc}
\hline \multicolumn{1}{c}{ Área da Fisioterapia } & \multicolumn{2}{c}{ Masculino } & \multicolumn{2}{c}{ Feminino } & Total \\
& $\mathbf{n}$ & $\mathbf{I M} \pm \mathbf{D P}$ & $\mathbf{n}$ & $\mathbf{I M \pm D P}$ & $\mathbf{M + F}(\%)$ \\
\hline Ortopedia-Funcional & 103 & $40 \pm 16,49$ & 80 & $48 \pm 15,16$ & $183(66,06 \%)$ \\
Neuro-Funcional & 37 & $47 \pm 26,14$ & 32 & $59 \pm 20,68$ & $69(28,51 \%)$ \\
Reumato-Funcional & 08 & $57 \pm 22,29$ & 09 & $60 \pm 11,82$ & $17(6,13 \%)$
\end{tabular}


Cardiorrespiratoria-

Funcional

Uroginecologia-

Funcional

Oncologia-Funcional Total
01

56

-

$14943 \pm 20,0$

05

$59 \pm 19,99$

37

$2(0,72 \%)$

Legenda: IM: Idade Média; DP- Desvio Padrão; N; número amostral; M: Masculino; F: Feminino.

Fonte: Elaborado pelos autores (2021)

A tabela 02 mostra os números absolutos e percentuais ( $\mathrm{N}-\%)$ dos principais diagnósticos clínicos que levaram os pacientes a buscar por atendimentos no setor de fisioterapia, comparando os sexos. Conforme os dados da referida tabela, a área de ortopedia-funcional apresentou a maior busca pelo serviço de fisioterapia sendo que o diagnóstico de fraturas ósseas foi o mais presente, tanto para o masculino como feminino ( $M=20,57 \%$ e $F=11,91 \%)$. A segunda especialidade com maior de procura foi a neuro-funcional e, neste caso, o diagnóstico de acidente vascular encefálico ( $M=18-6,49 \%$ e $F=9,02 \%$ ) foi o mais encontrado. Para a especialidade da reumatofuncional o diagnóstico de artrose foi o mais presente $(\mathrm{M}=2,16 \%$ e $\mathrm{F}=2,52 \%)$. As áreas com menores procuras foram cardiorrespiratórias, uroginecologia e oncologia, com valores aproximados de $1 \%$ para cada área.

Tabela 2. Distribuição de pacientes considerando áreas de atuação da fisioterapia, o diagnóstico clínico e o sexo.

\begin{tabular}{cccc}
\hline Área & Diagnóstico & Masculino (n- \%) & Feminino (n- \%) \\
\hline & Fratura & $57-20,57 \%$ & $33-11,91 \%$ \\
\cline { 2 - 4 } $\begin{array}{c}\text { Ortopedia- } \\
\text { funcional } \\
(\mathbf{n}=\mathbf{1 8 3})\end{array}$ & $\begin{array}{c}\text { Lesão Musculo- } \\
\text { tendínea }\end{array}$ & $25-9,02 \%$ & $26-9,38 \%$ \\
\cline { 2 - 4 } & Lesão Ligamentar & $10-3,61 \%$ & $04-1,44 \%$ \\
\cline { 2 - 4 } & Disfunção na Coluna & $09-3,24 \%$ & $09-3,24 \%$ \\
\cline { 2 - 4 } & Lesão Meniscal & $05-1,80 \%$ & $00-0$ \\
\hline \multirow{2}{*}{$\begin{array}{c}\text { Neuro- } \\
\text { funcional } \\
(\mathbf{n}=\mathbf{7 9})\end{array}$} & Outros & $03-1,08 \%$ & $02-0,72 \%$ \\
\cline { 2 - 4 } & AVE & $18-6,49 \%$ & $25-9,02 \%$ \\
\cline { 2 - 4 } & TCE & $04-1,44 \%$ & $00-0$ \\
\hline
\end{tabular}




\begin{tabular}{|c|c|c|c|}
\hline \multirow{3}{*}{$\begin{array}{l}\text { Reumato- } \\
\text { funcional } \\
(n=17)\end{array}$} & Artrose & $06-2,16 \%$ & $07-2,52 \%$ \\
\hline & Artrite & $01-0,36 \%$ & $01-0,36 \%$ \\
\hline & Outros & $00-0$ & $02-0,72 \%$ \\
\hline \multirow{3}{*}{$\begin{array}{c}\text { Demais áreas } \\
\qquad(\mathrm{N}=8)\end{array}$} & $\begin{array}{c}\text { Cardiorrespiratória } \\
\text { (DPOC, COVID-19, } \\
\text { derrame pleural, ponte } \\
\text { safena) }\end{array}$ & $00-0$ & $05-1,80 \%$ \\
\hline & Oncologia, linfoma & $00-0$ & $01-0,36 \%$ \\
\hline & $\begin{array}{l}\text { Urologia (perineal, dor } \\
\text { retal) }\end{array}$ & $01-0,36 \%$ & $01-0,36 \%$ \\
\hline
\end{tabular}

Legenda: AVE- Acidente vascular encefálico; TCE - Traumatismo Crânio-encefálico; DPOC - Doença Pulmonar obstrutiva Crônica; COVID-19 - Doença causada pelo novo Coronavírus. Fonte: Elaborado pelos autores (2021).

$\mathrm{Na}$ tabela 3 apresentam-se os resultados referentes ao número de terapias realizadas e faltas. Conforme os dados, os pacientes de reumato-funcional foram os que mais receberam terapias com a média de 13 atendimentos, seguido pelos pacientes da ortopedia-funcional e neuro-funcional com média de 10 atendimentos cada. Para as demais áreas, que incluiu cardiorrespiratória, uroginecologia e oncologia funcional, observou-se média de 4 terapias. Em relação ao número de faltas os pacientes de neuro-funcional tiveram 2 faltas, em média. Já na ortopedia-funcional e reumato-funcional tiveram, em média, uma falta cada. As demais áreas agrupadas não apresentaram faltas.

Tabela 3. Média de Terapias e Faltas para cada Área de atuação da fisioterapia

\begin{tabular}{ccc}
\hline Áreas & Média de Terapias & Média de Faltas \\
\hline Reumato-funcional & 13 & 1 \\
Ortopedia-funcional & 10 & 1 \\
Neuro-funcional & 10 & 2 \\
Demais Áreas & 4 & - \\
\hline
\end{tabular}

Fonte: Elaborado pelos autores (2021).

\section{DISCUSSÃo}

O presente estudo surgiu com o intuito de descrever o perfil dos pacientes atendidos em um serviço municipal de reabilitação da região metropolitana de Curitiba/PR. Estudos desta magnitude auxiliam a gerar conhecimento, a tomada de decisão, formulação de políticas e/ou organização do sistema de saúde e, ainda, 
propor intervenções destinadas a dar solução aos problemas específicos em relação ao tipo de pacientes que frequentam o setor. Desta forma, conhecendo estes dados, os gestores municipais e os profissionais do setor podem desenvolver protocolos específicos que fortaleçam o serviço e sejam mais resolutivos. Por outro lado, os dados do presente estudo também podem conscientizar os usuários do serviço público de saúde por mostrar de forma fidedigna as não aderências ao tratamento (faltas constantes / abandono sem aviso, entre outras), o que limita os profissionais a chamarem novos pacientes por trabalharem com a agenda fechada. Portanto, os dados epidemiológicos do presente estudo têm um papel importante e direto para a saúde pública.

Observando os dados da presente pesquisa, percebeu-se que a maioria dos pacientes atendidos no setor de fisioterapia do município foi do sexo masculino $(56 \%)$, tanto em 2019 como em 2020. Estes dados divergem de um estudo anterior realizado no Estado de Goiás e que avaliou 59 prontuários e a maioria (34) correspondiam a indivíduos do sexo feminino e a minoria (25) a indivíduos do sexo masculino ${ }^{1}$. Já em outro estudo realizado em uma cidade do Estado de São Paulo, observou-se que em relação ao gênero, $866(57,46 \%)$ pacientes eram do sexo masculino e 641 (42,54\%) do sexo feminino ${ }^{13}$. Isso mostra que os perfis podem variar em relação aos períodos temporais e/ou regiões geográficas. Por isso, atualizar constantemente o perfil da população atendida é de interesse dos profissionais da área da saúde, uma vez que possibilita investigar a raiz do problema e determinar a real situação de saúde da população.

Constatou-se no presente estudo que a média de idade foi de $43 \pm 20,0$ anos para o gênero masculino e $52 \pm 17,2$ anos para o feminino. Em outro estudo, realizado em outra região mostrou que a maior prevalência foi na faixa etária acima de 60 anos $(55,6 \%)^{14}$. Importante destacar que, para o presente estudo, algumas áreas apresentaram apenas um paciente atendido e, por isso, infere-se que a idade apresentada não é de fato representativa.

A presente pesquisa também investigou a área de maior busca pela fisioterapia. Sobre este resultado, a maior demanda deu-se na orto-funcional $(66,06 \%)$ sendo que os diagnósticos clínicos mais comuns foram fraturas, lesões músculo-tendíneas ou ligamentares, disfunções na coluna e lesões meniscais. Outros diagnósticos como 
pós-operatório de prótese de quadril, amputação de membro e derrame articular também foram atendidos, mas em menor quantidade. Por isso, foram agrupados para análise no presente trabalho. As fraturas foram em maior número no sexo masculino. Este achado corrobora com outra linha investigativa que também demonstrou o predomínio de fraturas em indivíduos do sexo masculino, representando $65,4 \%$ dos $\operatorname{casos}^{15}$. Outro estudo realizado no estado de Alagoas também relatou que a área com maior procura foi a traumatologia, porém o diagnóstico mais frequente foi póscirúrgico ${ }^{16}$.

Embora não tenha sido o objetivo do estudo, percebeu-se que as causas do alto índice de pacientes com diagnóstico de fratura decorrem dos acidentes automobilísticos ou quedas, tanto de nível quanto de altura. Este achado está de acordo com as descrições que concordam entre si que as fraturas por queda de nível são decorrentes de baixa energia e mais predominantes em idosos, e os traumas por queda de altura, e acidentes automobilísticos estão associados aos indivíduos mais jovens ${ }^{17}$. Em conversa com os profissionais, os pesquisadores tiveram como possível justificativa do alto número de acidentes o fato do município estar situado aproximadamente 30 quilômetros de Curitiba e a maioria da população ter a necessidade de se deslocar até capital para trabalhar. Desta forma, o índice de acidentes na rodovia que liga as duas cidades é altíssimo.

$\mathrm{Na}$ área de neuro-funcional as doenças de maior incidência foram acidente vascular encefálico (AVE) e traumatismo craniano (TCE). Outras patologias como Parkinson, Paralisia Cerebral, Meningite, Esclerose Lateral Amiotrófica, Poliomielite, Lesão Medular, Paralisia de Bell, Mieolopatia, Polineuropatia e atraso no desenvolvimento psicomotor também foram atendidas, porém em menor número. $\mathrm{O}$ maior índice de AVE atendidos no setor pode ser explicado pelo fato desta ser uma consequência de hábitos de vida não saudáveis e, uma vez que a pessoa tenha sido acometida, tanto o paciente como a família terão um impacto pela ocorrência de sequelas motoras e neurológicas que levam a incapacidade funcional o que aumenta a busca pelos serviços de reabilitação ${ }^{18}$.

Ainda sobre a prevalência do AVE, percebeu-se que esta patologia foi mais comum nas mulheres. Este achado confronta com outros autores que citam a tendência de homens serem mais acometidos ${ }^{19}$. Por outro lado, a maior ocorrência do 
AVE no sexo feminino pode ser decorrente de alguns fatores específicos como alterações hormonais, gestação, uso de anticoncepcionais e/ou depressão ${ }^{20}$. Além disso, a predominância feminina nas taxas de expectativa de vida é maior e, conforme a idade avança, os riscos de acometimento de doenças cerebrovasculares são maiores $^{20}$. Desta forma, sugere-se a formulação de políticas públicas que fortaleçam a promoção a saúde e que permita maior atuação do fisioterapeuta na atenção primária, desenvolvendo campanhas específicas para prevenção de doenças crônicas degenerativas. Atualmente a atuação do fisioterapeuta é mais voltada para a reabilitação.

Sobre os acometimentos de TCE, segunda causa neurológica de maior procura pelo serviço de fisioterapia, percebeu-se que os pacientes eram sempre do sexo masculino. O TCE é mais prevalente no sexo masculino devido ao comportamento humano dos indivíduos deste sexo, o qual tendem apresentar um ritmo de vida mais agressivo, imprudente, por viver de emoções e favorecer situações que os colocam em perigo ${ }^{20-21}$. Mediante os achados do presente estudo e considerando as descrições da literatura, nota-se que os gestores públicos de saúde devem buscar parcerias com outros órgãos públicos para elaborar campanhas educativas que visem à diminuição da violência urbana. Além disso, ações educativas sobre o trânsito também se fazem necessárias para diminuir o número de pessoas lesionadas, não apenas com TCE, mas também abrangendo outras lesões, como Lesão medular, fraturas, dentre outras. Percebe-se que o setor de saúde é um gargalo da falta de outras políticas públicas.

Sobre os achados referente as doenças reumáticas que são as principais doenças não transmissíveis no país e que as literaturas mostram que os maiores acometimentos ocorrem no sexo feminino ${ }^{22-23}$ e a amostras obtidas na atual pesquisa também apresentou o maior índice destas doenças neste sexo.

O resultado deste trabalho também mostrou que algumas patologias possuem baixo índice de procura no setor pesquisado, tais como as áreas cardiorrespiratóriafuncional, uroginecologia-funcional e oncologia-funcional. A fisioterapia possui um papel fundamental na intervenção contra a covid-19, desde sintomas leves, no âmbito hospitalar e pós-alta. Dessa forma, durante a pandemia houve um grande crescimento na necessidade de fisioterapeutas respiratórios. No entanto, o setor em que a 
pesquisa foi realizada não houve um aumento pois o município ainda estava apresentando poucos casos ${ }^{24}$.

Outro ponto importante a discutir trata-se da redução do número de pacientes atendidos entre os anos de 2019 e 2020. A diferença ocorreu devido a pandemia de SARS-CoV-2 que afetou não somente o setor da saúde, mas a sociedade em geral. Dada a natureza complexa da resposta às emergências de saúde pública, nas quais os limites e a resiliência do sistema de saúde foram colocados à prova ${ }^{25}$, a Secretaria Municipal de Saúde do município de realização desta pesquisa optou por alguns mecanismos específicos relacionados ao combate a pandemia, o que incluiu a reorganização e/ou cancelamento dos atendimentos eletivos. Estas medidas foram necessárias mediante a emergência da epidemia, os quais vários serviços foram suspensos ou tiveram produção reduzida visando reduzir aglomerações e a propagação do novo vírus. Assim, devido às incertezas da doença, os serviços de saúde também reduziram o atendimento pelo risco de contágio e precisaram se adaptar para atender pacientes com a síndrome respiratória, sendo repensado o modelo convencional de atenção, focando em reduzir a propagação da contaminação e analisando caso a caso dos pacientes para não promover prejuízo aos tratamentos 26 .

Neste cenário, no Centro Municipal de reabilitação não foi diferente. Profissionais relataram que o setor foi fechado por várias vezes durante o ano de 2020 pelo fato de alguns profissionais testarem positivos ou ficarem isolados preventivamente. Além disso, pela ausência de profissionais em outros setores, os fisioterapeutas eram realocados em diferentes funções da secretaria municipal de saúde para substituir pessoas que eram afastadas/isoladas. Todos estes fatores culminaram em uma redução do número de pacientes atendidos em aproximadamente $58 \%$, comparando os dois anos $(2019=224 ; 2020=95)$. Tal redução corrobora com outro estudo realizado em São Paulo, o qual mostrou declínio de 43\% nos atendimentos eletivos de consultas médicas e de $42,2 \%$ no número de exames especializados no período da pandemia da COVID-19 26.

Vale destacar, ainda, que houve notas orientativas e um decreto Municipal que permitia aos servidores que se enquadrassem em grupos de riscos a realizar atividades home-office visando proteger a própria saúde destes trabalhadores e de 
seus familiares. Desta forma, no CMR duas fisioterapeutas foram direcionadas a realizar o trabalho desta forma: uma por apresentar doença relacionada a imunidade e outra por estar gestante. Inicialmente, estas trabalhadoras realizavam serviços administrativos, também impactando na redução de novos atendimentos.

Embora não tenha sido o objetivo da presente pesquisa, notou-se que durante o auge da pandemia, os fisioterapeutas foram chamados para compor atendimentos no hospital Municipal o qual foi estruturado para dar suporte aos pacientes internados por COVID-19. Por estes fatores, o tratamento de pacientes com COVID-19 foi um grande desafio para a equipe de Fisioterapia devido à necessidade de pessoal com formação em ventilação mecânica e equipamentos adicionais, imprescindíveis para atender ao grande aumento de pacientes internados em um curto espaço de tempo ${ }^{27}$. Assim, como continuidade do estudo, sugere-se realizar uma nova coleta de dados observando o perfil epidemiológico e funcional dos pacientes que foram encaminhados ao setor de fisioterapia no ano de 2021 e/ou que foram acometidos pelo SARS-CoV-2.

Uma das limitações do presente estudo é a falta de coleta de dados de ocupação/profissão, escolaridade, raça e fatores de riscos dos pacientes envolvidos. Estas informações não foram consideradas por não constar em todos os prontuários. Em novas investigações, sugere-se incluir estes itens para melhor correlacionar os dados.

\section{CONCLUSÃO}

Com base nas análises e resultados foi possível traçar o perfil epidemiológico dos pacientes da fisioterapia de um município da região metropolitana de Curitiba durante os anos de 2019 e 2020. Evidenciou-se que o sexo mais prevalente no setor de fisioterapia foi o masculino (56\%) e a idade média geral foi de $43 \pm 20,0$ e $52 \pm 17,2$ anos para o sexo masculino e feminino, respectivamente. A área da orto-funcional, o diagnóstico de fraturas ósseas foi mais presente. Na neuro-funcional, o AVE apresentou maior demanda e, na reumato-funcional, o diagnóstico de artrose foi mais recorrente. Apesar da possibilidade de traçar o perfil epidemiológico, ainda são necessários novos estudos para que se estabeleçam protocolos avançados de atendimento em diferentes grupos de doenças. 


\section{REFERÊNCIAS}

1. Silva PHB, Lima KA, Leroy PLA. Perfil epidemiológico dos pacientes assistidos na clínica de Fisioterapia Traumato-ortopédica da Prefeitura de Hidrolândia - Goiás. Movimenta [Internet]. 28fev.2018 [citado 23ago.2021]; 6(3):520-9. Disponível em: https://www.revista.ueg.br/index.php/movimenta/article/view/7015

2. Hartz ZMA, Contandriopoulos, AP. Integralidade da atenção e integração de serviços de saúde: desafios para avaliar a implantação de um "sistema sem muros". Cad. Saúde Pública [online]. 2004, v. 20, suppl2 [Acessado 23 Agosto 2021], pp. S331-S336. doi: https://doi.org/10.1590/S0102-311X2004000800026

3. Albuquerque MAL, Carvalho VCP. O papel do fisioterapeuta no Programa Saúde da Família. Revista Inspirar [Internet]. 2009 [Acesso em 13 Julho 2021]; 1(2):15-9. Disponível em: http://www.inspirar.com.br/revista/wpcontent/uploads/2010/04/revista cientifica i nspirar edicao 2 2009.pdf

4. Mendes EV. As redes de atenção à saúde. Divergência no diagnóstico. Ciênc. saúde coletiva. [Internet]. 2010 [acesso em 07 dezembro 2021]. 15(5) doi: https://doi.org/10.1590/S1413-81232010000500005

5. Maciel RV, Silva PTG, Sampaio RF, Drummond AF. Teoria, prática e Realidade Social: uma Perspectiva Integrada para o Ensino de Fisioterapia. Fisioter. Mov. [Internet]. 2005 [acesso em 23 Agostos 2021]; 18(1). Disponível em: https://periodicos.pucpr.br/index.php/fisio/article/view/18522

6. Ribeiro CD, Flores-Soares MC. Desafios para a inserção do fisioterapeuta na atenção básica: o olhar dos gestores. Rev. salud pública [online]. 2015 [acesso em 29 outubro 2021]; 17(3). doi: https://doi.org/10.15446/rsap.v17n3.44076

7. Bispo Júnior JP. Fisioterapia e Saúde Coletiva: Desafios e Novas Responsabilidades Profissionais. Ciênc. Saúde Colet. [Internet]. 2010. [acesso em 30 agosto 2021]; 15(1). doi: https://doi.org/10.1590/S1413-81232010000700074

8. Couto C, Ribeiro FC, Barradas NA, Matos M, Coelho E, Luzes R. Perfil Epidemiológico dos Atendimentos Realizados na Clínica Escola de Fisioterapia da UNIABEL. Revista Saúde Física \& Mental- UNIABEL [internet]. 2014 [acesso em 29 Agosto 2021]; 4(1). Disponível em: https://revista.uniabeu.edu.br/index.php/SFM/article/view/1791/1167

9. Santos FAS, Netto JSL, Ramos JCL, Soares FO. Perfil Epidemiológico dos atendidos pela fisioterapia pelo programa saúde e reabilitação na família em Camaragibe, PE. Fisioter. pesqui. [Internet]. 2007 [acesso em 29 Agosto 2021]; 14(3). doi: https://doi.org/10.1590/fpusp.v14i3.76111

10. Batista AJ, Torres JR, Schwind RTF, Peternella FN, Mendes FV. Perfil Epidemiológico do Setor de Neurologia da Clínica Escola de Fisioterapia da Faculdade Uningá no ano 2013. UningáReview [Internet]. 2014 [acesso em 28 Agosto 2021]; 17(6). Disponível em http://revista.uninga.br/index.php/uningareviews/article/view/1494/1109 
11. Gil; AC. Como Elaborar Projeto de Pesquisa. 4ª Edição. São Paulo: Atlas; 2008.

12. Instituto Brasileiro de Geografia e Estatística (IBGE). Cidades e Estados População Estimada [internet]. Brasil; 2021. Disponível em: https://www.ibge.gov.br/cidades-e-estados/pr/rio-branco-do-sul.html

13. Silva DV, Andrusaitis SF, Fernandes LG, Melo TB, Junior NC, Brech GC. Prevalência de idade e gênero e sua correspondência com os setores de fisioterapia ambulatorial de um instituto de ortopedia e traumatologia de referência da cidade de São Paulo. Fisioter Pesqui. [Internet] 2019 [acesso em 29 outubro 2021]; 26(4). doi: https://doi.org/10.1590/1809-2950/18026426042019

14. Menegazzo IR, Pereira MR, Villalba JP. Levantamento epidemiológico de doenças relacionadas à fisioterapia em uma Unidade Básica de Saúde do município de Campinas. J Health Sci Inst. [Internet] 2010 [acesso em 29 outubro 2021]; 28(4). Disponível em: content/uploads/2020/12/V28 n4 2010 p348-351.pdf

15. Court-Browm CM, Caeser B. Epidemiology of adult fractures: A Review. Injury [Internet]. 2006 [acesso em 30 Agosto 2021]; 37(8). doi: https://doi.org/10.1016/.injury.2006.04.130

16. Oliveira JC, Santos RPMC, Calles ACNC, Monteiro FT. Perfil Epidemiológico dos pacientes atendidos em uma Clínica-Escola de Fisioterapia na Cidade de MaceióAL. Interfaces Científicas - Saúde e Ambiente. [internet]. 2018 [acesso em 29 outubro 2021]; 6(2). doi: https://doi.org/10.17564/2316-3798.2018v6n2p85-94

17. Souza JAG, Iglesias ACRG. Traumas no Idoso.AMB rev. Assoc. Med. Bras. [Internet]. 2002 [acesso em 30 Agosto 2021]; 48(1). doi: https://doi.org/10.1590/S0104-42302002000100037

18. Freitas EV, PY L.Cançado FA, Doll J, Gorzoni ML. Tratamento de Geriatria e Gerontologia. 3를 edição. Rio de Janeiro: Guanabara Koogan, 2013.

19. Mota E, Natalino MA, Waltrick, PT. Intervenção fisioterapêutica e tempo de internação em pacientes com Acidente Vascular Encefálico. Rev. neurociênc. [internet]. 2008 [acesso em 30 Agosto 2021]; 16(2). doi: https://doi.org/10.34024/rnc.2008.v16.8648

20. Gonçalves JL, Feitosa, ES, Borges, RT. Perfil epidemiológico de vítimas de acidente vascular encefálico em um hospital de referência do Ceará/Brasil. R. Interd. [Internet]. 2019 [acesso em 24 Agosto 2021]; 12(2). Disponível em: https://dialnet.unirioja.es/servlet/articulo?codigo $=7868624$

21. Farage L, Colares VS, Neto MC, Moraes MC, Barbosa MC, Branco Junior JA. As Medidas de Segurança no Transito e a Morbimortalidade Intrahospitalar por traumatismo cranioencefálico no distrito federal. AMB rev. Assoc. Med. Bras. [internet]. 2002 [acesso em 30 Agosto 2021]; 48(2). doi: https://doi.org/10.1590/S0104-42302002000200037

22. Vaz AE, Júnior WAF, Lazarski CFS, Carmo HF, Sobrinho HMR. Perfil epidemiológico e clínico de pacientes portadores de artrite reumatóide em um 
hospital escola de medicina em Goiânia, Goiás, Brasil. Medicina (Ribeirão Preto). [Internet]. 2013 [acesso em 29 Agosto 2021]; 46(2). doi: https://doi.org/10.11606/issn.2176-7262.v46i2p141-153

23. Malta DC, Stopa SR, Szwarcwald CL, Gomes NL, Júnior JBS, Reis AAC. A vigilância e o monitoramento das principais doenças crônicas não transmissíveis no Brasil - Pesquisa Nacional de Saúde. Rev. bras. epidemiol. [internet] 2015 [acesso em 29 Agosto 2021]; 18(2). doi: https://doi.org/10.1590/1980$\underline{5497201500060002}$

24. Dias CS, Camelier FWR, Santos MLM. Atuação dos fisioterapeutas no âmbito da Atenção Primária à Saúde (APS) junto a usuários suspeitos ou diagnosticados com COVID-19*: contribuições da Fisioterapia Respiratória. Assobrafir Ciência. [internet]. 2020 [acesso em 29 outubro 2021]; 11(supl 1). doi: http://dx.doi.org/10.47066/2177-9333.AC20.covid19.004

25. Pazin-Filho A, Jardim DB, Dallora MELV, et al. Gabinete de Crise do HCFMRPUSP como estratégia de gestão das demandas da crise de Pandemia COVID-19. Revista Qualidade HC - Revista Eletrônica. [internet]. 2021 [acesso em 07 dezembro 2021]; $\quad$ (1). Disponível em https://www.hcrp.usp.br/revistaqualidadehc/uploads/Artigos/448/448.pdf

26. Silva NCA, Moroço DM, Carneiro PS. O impacto da pandemia de COVID-19 no atendimento eletivo: experiência de um Hospital de nível terciário e Centro de Referência para a doença. Revista Qualidade HC - Revista Eletrônica. [internet]. 2021 [acesso em 07 dezembro 2021]; 2(1). Disponível em https://www.hcrp.usp.br/revistaqualidadehc/uploads/Artigos/447/447.pdf

27. Bottura C, Mazzoni AAS, Leite, MB, Hussein AM, Gastaldi AC. Atuação do serviço de fisioterapia nas unidades de terapia intensiva no contexto da pandemia de COVID-19. Revista Qualidade HC - Revista Eletrônica. [internet]. 2021 [acesso em 07 dezembro 2021]; 2(1). Disponível em https://www.hcrp.usp.br/revistaqualidadehc/uploads/Artigos/449/449.pdf

28. Tacon FSA, Moraes CL, Evangelista PG, Amaral WN, Amaral-Filho WN. Divergência no diagnóstico ultrassonográfico de malformações fetais entre nível primário e terciário do sistema única de saúde (SUS). Revista Brasileira de Ultrassonografia. [internet]. 2018 [acesso em 07 dezembro 2021]. 25 ed. Disponível em https://sbus.org.br/wp-content/uploads/2018/10/revistasetembro.pdf 